\title{
A Novel Topology for the Zonal Network with Wireless Coverage
}

\author{
Fumin ZOU ${ }^{1}$, Xinhua JIANG ${ }^{1}$, Zhangxi LIN ${ }^{2}$, Tongsen WANG ${ }^{1}$ \\ ${ }^{1}$ Research Center for Next-Generation Internet Tech \& Apps, Fujian University of Technology, Fuzhou, China \\ ${ }^{2}$ College of Business Administration, Texas Tech University, Lubbock, TX, USA \\ E-mail: fuminzou@ngi.fj.cn, zhangxi.lin@ttu.edu \\ Received August 5, 2009; accepted September 4, 2009
}

\begin{abstract}
In this paper, we propose a banyan-tree topology for the wireless coverage along the road or railway, which is characterized as a zonal network. Theoretical analysis and numerical studies show that the proposed banyan-tree topology is appropriate for the zonal network with the Wireless Mesh Network (WMN) technology, which is feasible to deploy and has the enhanced robust, improved bandwidth with this topology.
\end{abstract}

Keywords: Banyan-Tree Topology, Zonal Network, Wireless Coverage, Transportation

\section{Introduction}

While the population of Internet users in China has reached 338 millions by June 2009 [1], 26.8 billion passengers on road [2] and 1.46 billion on railway [3] spend billions of unproductive hours during their traveling, showing that it is not only the demand to promote the transportation service quality, but also a great potential market for the Internet services on the fleeting car [4]. As well as the Car-Ground Internetworking (CGI), the Zonal Wireless Coverage (ZWC) is another essential infrastructure for the onboard Internet applications.

For the ZWC, WiFi is an appropriate technology that is technically and economically feasible, and has attracted substantial attention in the wireless and transportation communities [5-8]. However, deploying hundreds even thousands of APs along the road or railway is a main challenge to this promising technology because of the limited coverage. So far, several topologies have been put into trial implementation for the ZWC with WiFi. Among them, the star topology was found almost infeasible since too many cables are require, and the bus topology is also infeasible because the multi-hop Wireless Mesh Network (WMN) is too weak $[9,10]$.

This paper proposes a novel topology for the deployment of WiFi in the ZWC context. It is claimed that the WMN is feasible to implement the ZWC with fewer cables to deploy and its robust can be promoted to some extent by splitting the multi-hop WMN to multiple sectors [5]. However, if a mesh node failed, its subsequent nodes in the same chain will become invalid too. As an improvement of the splitting scheme, our approach lets the packets to be forwarded via a most reliable path in the zonal network, in which all the nodes are connected one by one with wireless link, while a number of portals are deployed to the wired network. To avoid the circle and get the most reliable path, we designed a Dynamical Minimal Spanning Tree (DMST) algorithm, which will update the forwarding path periodically according to the dynamical changing fault probability. Hence, the packets can be forwarded via another path to its destination automatically once a mesh node failure.

To overcome the bandwidth problem which is reported in references [11,12], i.e., the bandwidth dramatically decreased as $1 / \mathrm{n}$ even $1 / 2^{n}$ in multi-hop WMN, the multi-interface mesh node structure is introduced and it is found that the banyan-tree topology has an improved bandwidth in ZWC.

The outline of this paper is as follows. In Section 2, we present the banyan-tree topology. The algorithm DMST is designed in Section 3, and the network robust, bandwidth and etc are analyzed in Sections 4 and 5. Section 6 presents outcomes from the numeric study, and this paper is concluded by Section 7 .

\section{Banyan-Tree Topology}

Definition 1: Let $G=(V, E)$ be a connected graph with $|V|$ $=\mathrm{n}+1$ nodes, if

1) $G$ has a Hamiltonian cycle $v_{0}, v_{1}, v_{2}, \cdots, v_{n}, v_{0}$;

2) $\mathrm{G}$ at least has a node $v_{j}$ connecting $v_{0}$ except $v_{1}$ and $v_{n}$.

Then we call graph $\mathrm{G}$ as a banyan-tree topology. 
Where $v_{0}$ is called the root and its neighbors are called prop-nodes. Let $V^{*}$ be the order set of prop-nodes, if $\left|V^{*}\right|=n$ then it is a star topology, else if $\left|V^{*}\right|=1$ then it is a bus topology. Figure 1 is an example of banyan-tree topology.

Apparently, the banyan-tree topology contains a number of circles which should be avoided in data communication that requires the packets forwarded along a certain minimal spanning tree. Hence, let us draw a weighted graph $\mathrm{G}=(\mathrm{V}, \mathrm{E}, \mathrm{W})$ by assigning a cost to each edge, where $\mathrm{W}$ is the set of weights. Then the best forward path can be computed by a minimal spanning tree algorithm.

Let the danger coefficient of each edge be its cost, and give the related definitions as follows.

Definition 2: Let $v_{j} \in V^{*}$, if $c<i, j>=v_{i}, \cdots, v_{j}$ is a maximal path extended from $v_{j}$, where $v_{0} \notin c<i, j>\wedge v_{i} \notin V^{*}$, then $c<i, j>$ is called a multi-hop chain.

Obviously, for every node $v_{j} \in\left\{v_{2}, \cdots, v_{n-1}\right\}$ it has $v_{j} \in c<i, j>\wedge v_{j} \in c<j, k>$, where $v_{i}=v^{x} \wedge v_{k}=v^{x+1}$.

Definition 3: for every node $\left.v_{k} \in c<i, j\right\rangle$, we denote $O_{k}=1-\prod_{t=j}^{k}\left(1-o_{t}\right), i \leq k \leq j \quad$ as its partially invalidated probability (PIP), where $o_{t}$ is the fault probability of each node in the chain.

Let us see the example of Figure 1. If $o_{t}=1 \%$ for any $v_{t} \in c<5,8>$ then $O_{6}=2.997 \%$ according to the Definition 3. Hence this definition is reasonable because if any node of $\left\{v_{6}, v_{7}, v_{8}\right\}$ failures, the packets from $v_{6}$ cannot reach $v_{0}$ via this path.

Since a node $v_{k}$ is associated to two multi-hop chains, it has two PIPs, say $\left(O_{k}^{1}, O_{k}^{2}\right)$. Then its completely invalidated probability (CIP) in the banyan-tree topology is $O_{k}^{1} \times O_{k}^{2}$. But for the minimal spanning tree every node will belong to a certain multi-hop chain, hence we define the danger coefficient as follows.

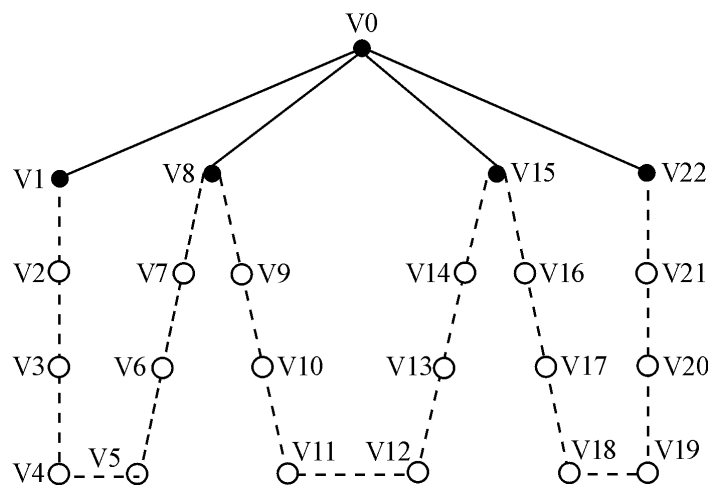

Figure 1. Example of banyan-tree topology.
Definition 4: Denote the pair of PIPs of $v_{k}$ in its left and right multi-hop chain as $\left(O_{k}^{1}, O_{k}^{2}\right)$, and denote its danger coefficient as $E_{k}=\min \left(O_{k}^{1}, O_{k}^{2}\right)$.

To simplify, we assume that danger coefficients of both $v_{0}$ and wired network are 0 , and the danger coefficient of edge $\left\langle v_{k}, v_{k+1}\right\rangle$ is equal to $E_{k}$. These imply that our discussion is based on the condition when $v_{0}$ and the wired network are running normally, and the state of the wireless link is determined by the mesh node. With these assumptions, let the fault probability of each node be $1 \%$ except $v_{0}$, then the spanning tree of Figure 1 with the minimal invalid risk can be shown in Figure 2.

\section{Dynamical Minimal Spanning Tree Algorithm}

When a mesh node failures, packets should be automatically forwarded via the alternative path to its destination. Furthermore, when a node's fault probability is varying in accordance with time, the ZWC should be able to dynamically find a more reliable path to forward the packets. Assume that the fault probability vector is $\left(o_{1}, o_{2}, \cdots, o_{n}\right)$ except $v_{0}$ at time $t$, the algorithm DMST is designed as follow.

Dynamical Minimal Spanning Tree Algorithm $\left(o_{1}, o_{2}, \cdots, o_{n}\right)$

\{

Step 1: Let order set $V^{*}=\left\{v_{1}^{1}, v_{i}^{2}, v_{j}^{3}, \cdots, v_{n}^{m}\right\}$, then its danger coefficient vector $\left(E_{1}, E_{i}, E_{j}, \cdots, E_{n}\right)=\left(o_{1}, o_{i}, o_{j}, \cdots, o_{n}\right)$.

Step 2: Let order set $\left\{c_{1}^{1}, c_{i}^{2}, c_{i}^{3}, c_{j}^{4}, c_{j}^{5} \cdots, c_{n}^{2 m-2}\right\}$ be the multi-hop chains terminated by the prop-nodes, then its initial no-failure probability vector $\left(O c_{1}^{1}, O c_{i}^{2}, O c_{i}^{3}, O c_{j}^{4}, O c_{j}^{5} \cdots, O c_{n}^{2 m-2}\right)$ $=\left(1-E_{1}, 1-E_{i}, 1-E_{i}, 1-E_{j}, 1-E_{j}, \cdots, 1-E_{n}\right)$.

Step 3: Partition root $v_{0}$, the prop-nodes $\left(v_{1}^{1}, v_{i}^{2}, v_{j}^{3}, \cdots, v_{n}^{m}\right)$ and their related edges to directed graph $G^{\prime}$ as the initial multi-hop chains.

Step 4: Loop While graph $G$ has not terminated multi-hop chain $C_{k}$.

Step 5: Add $v_{s}$ to $C_{k}$ to extend the path.

Step 5.1: if $v_{s}$ is not selected and $o_{s} \neq 1$ then go to Step 6.

Step 5.2: else terminate $C_{k}$ and go to Step 7 .

Step 6: Add $V_{s}$ and its edge to $C_{k}$, and update

$E c_{k}=E_{K} *\left(1-o_{s}\right), E_{K}=1-E c_{k}$.

Step 7: Select the next not terminated multi-hop chain.

Step 8: End of Loop While.

\} 


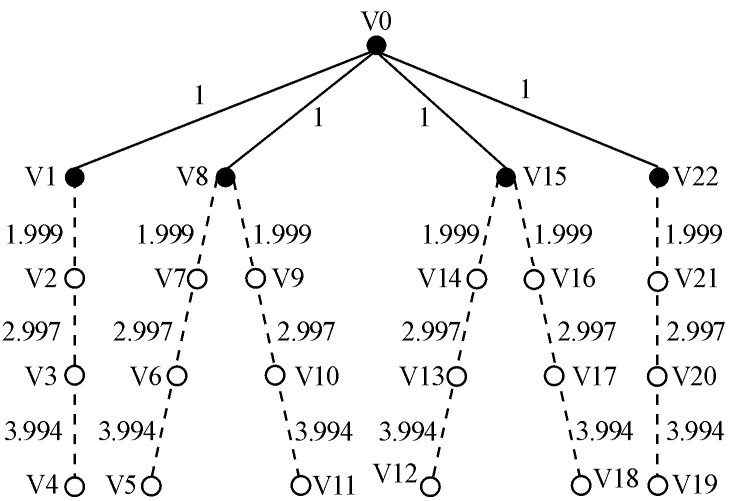

Figure 2. Spanning tree of the banyan-tree topology.

Graph $G^{\prime}$ is the minimal spanning tree according to the time-dependent fault probability.

Proposition 1: The time complexity of Algorithm DMST is $\mathrm{O}(|\mathrm{V}|+|\mathrm{E}|)$.

Proof. Assume that graph $\mathrm{G}$ is a banyan-tree topology. Then $\forall v_{k} \in V(G), v_{k} \neq v_{0}, \quad v_{k}$ belongs to a multi-hop chain $c<i, j>$ and $O_{k} \leq O_{t}, i \leq t \leq k$, according to Definition 3 and 4 . Hence the algorithm DMST only need do a breadth-first search (BFS) for graph $G$ to get the spanning tree with minimal invalid risk.

The time complexity of BFS algorithm for graph $\mathrm{G}$ is $\mathrm{O}(|\mathrm{V}|+|\mathrm{E}|)[13]$. Hence, the time complexity of algorithm DMST is $\mathrm{O}(|\mathrm{V}|+|\mathrm{E}|)$. Q. E. D.

\section{Network Robust Analysis}

Now we analyze the network robust of banyan-tree topology.

Proposition 2: The vertex connectivity of banyan-tree topology $\Psi=2$.

Proof. Assume that graph $\mathrm{G}$ is a banyan-tree topology. Then $\mathrm{G}$ has a Hamiltonian cycle $c=v_{0}, v_{1}, v_{2}, v_{3}, \cdots, v_{n}, v_{0}$ according to Definition 1. This means that $\forall v_{i} \in V(G)$, the number of connected components $p(G)=p\left(G-v_{i}\right)=1$, hence $\Psi \geq 2$. But when two nodes $v_{i}$ and $v_{j}$ ( $\left.v_{k} \notin V^{*}, 0<i \leq k \leq j\right)$ failures, $p\left(G-\left\{v_{i}, v_{j}\right\}\right)=2$, hence $\Psi \leq 2$.

Therefore, the vertex connectivity of banyan-tree topology $\Psi=2$. Q. E. D.

The network robust can be evaluated by the vertex connectivity for the traditional network. But for the ZWC with multi-hop WMN technology, the impact level of fault nodes must be assessed because a fault node always undertakes the task of packet relay for other nodes.

Definition 5: Denote the banyan-tree topology $G=(V, E)$ and the order set of fault nodes $V^{\prime}=\left\{v_{1}^{\prime}, v_{2}^{\prime}, \cdots, v_{m}^{\prime}\right\}, v_{0} \notin V^{\prime}$. Assume that $G^{\prime}$ is the largest connected components which are connected with $v_{0}$ when the fault nodes are deleted, i.e., $G^{\prime}=G\left(V-V^{\prime}, E\right)_{v_{0}}$, then $\alpha=\frac{|V(G)|-\left|V\left(G^{\prime}\right)\right|}{\left|V^{\prime}\right|}$ is the impact factor and $\Theta=|V(G)|-\left|V\left(G^{\prime}\right)\right|$ is the number of invalidated nodes.

The non-fault nodes in the isolated components which are disconnected from $v_{0}$ will become invalid, when the graph $\mathrm{G}$ becoming a disconnected graph affected by fault nodes. Hence defined as the ratio of the invalided nodes to fault nodes, the impact factor is appropriate to evaluate the network robust.

Proposition 3: Let the graph $\mathrm{G}=(\mathrm{V}, \mathrm{E})$ be a banyan-tree topology, where $V=\left\{v_{0}, v_{1}, v_{2}, \cdots, v_{n}\right\}$, the order set of fault nodes $V^{\prime}=\left\{v_{1}^{\prime}, v_{2}^{\prime}, \cdots, v_{m}^{\prime}\right\}, v_{0} \notin V^{\prime}$, and the order set of prop-nodes $V^{*}=\left\{v_{1}^{*}, v_{2}^{*}, \cdots, v_{l}^{*}\right\}$. If

$$
\begin{gathered}
\forall v_{i}^{\prime} \forall v_{k}^{*}\left(v_{i}^{\prime} \in L\left(v_{k}^{*}, v_{k+1}^{*}\right) \rightarrow \neg \exists v_{j}^{\prime}\left(v_{j}^{\prime} \in L\left(v_{k}^{*}, v_{k+1}^{*}\right)\right)\right. \\
\left.\vee \exists v_{j}^{\prime}\left(v_{j}^{\prime} \in L\left(v_{k}^{*}, v_{k+1}^{*}\right) \wedge\left(v_{i}^{\prime}, v_{j}^{\prime}\right) \in E\right)\right)
\end{gathered}
$$

then the impact factor $\alpha=1$.

Where $L\left(v_{k}^{*}, v_{k+1}^{*}\right)$ are the nodes of the shortest path which connects prop-node $v_{k}^{*}$ to $v_{k+1}^{*}$ and except $v_{0}$.

Proof. According to (1), given a banyan-tree topology $\mathrm{G}=(\mathrm{V}, \mathrm{E})$, for any circle $C_{k}=v_{0}+L\left(v_{k}^{*}, v_{k+1}^{*}\right)$ of $\mathrm{G}, C_{k}$ either has only one node or several adjacent nodes failure . Hence the nodes in $C_{k}$ are still connected when the fault nodes are deleted. In this way, $G^{\prime}=G-V^{\prime}$ is a connected graph for the arbitrariness of $C_{k}$.

Therefore we have $\alpha=\frac{|V(G)|-\left|V\left(G^{\prime}\right)\right|}{\left|V^{\prime}\right|}=\frac{\left|V^{\prime}\right|}{\left|V^{\prime}\right|}=1 \quad$ according to Definition 5. Q. E. D.

Let graph $\mathrm{G}=(\mathrm{V}, \mathrm{E})$ be a banyan-tree topology, the Factor $\alpha$ Algorithm is designed as follows for any order set of fault nodes $V^{\prime}=\left\{v_{1}^{\prime}, v_{2}^{\prime}, \cdots, v_{m}^{\prime}\right\}, v_{0} \notin V^{\prime} \wedge V^{\prime} \subset V$.

Procedure Factor $\alpha \operatorname{Algorithm}\left(v_{1}^{\prime}, v_{2}^{\prime}, \cdots, v_{m}^{\prime}\right)\{$

Step 1: Let nodes $V=\left\{v_{0}, v_{1}, v_{2}, \cdots, v_{n}\right\}$ and the order set of prop-nodes $V^{*}=\left(v_{1}^{*}, v_{2}^{*}, \cdots, v_{l}^{*}\right)$. Initialize the number of fault and invalid nodes with $\Omega=0, \Theta=0$.

Step 2: Loop While !Empty $\left(V^{*}\right)$.

Step 3: popup a fault node $V_{i}, \Omega++$.

Step 3.1: if $v_{i}^{\prime} \in L\left(v_{k}^{*}, v_{k+1}^{*}\right) \wedge v_{i+1}^{\prime} \notin L\left(v_{k}^{*}, v_{k+1}^{*}\right)$ or $v_{i}^{\prime} \in L\left(v_{k}^{*}, v_{k+1}^{*}\right) \wedge v_{i+1}^{\prime} \in L\left(v_{k}^{*}, v_{k+1}^{*}\right) \wedge\left(v_{i}^{\prime}, v_{i+1}^{\prime}\right) \in E$ then $\Theta++$.

Step 3.2: else $\Theta=\Theta+D\left(v_{i}^{\prime}, v_{i+1}^{\prime}\right)$.

Step 4: End of Loop.

Step 5: The impact factor $\alpha=\frac{\Theta}{\Omega}$. 
where $D\left(v_{i}^{\prime}, v_{i+1}^{\prime}\right)$ is the length of the shortest path $L\left(v_{i}^{\prime}, v_{i+1}^{\prime}\right)$, which connects fault node $v_{i}^{\prime}$ to $v_{i+1}^{\prime}$ except $v_{0}$

\section{Bandwidth and Latency Analysis}

Because the node in multi-hop WMN is not only need to provide the access for wireless users, but to undertake the task of packet relay for the left and right neighbors, the bandwidth will be decreased dramatically while the multihop chain increasing. Hence a multi-interface mesh node structure is presented as Figure 3.

The mesh node has 1 wired interface and 3 wireless interfaces, which is in charge of the wireless access, ingress and egress packet relay individually. When it acts as a prop-node, the traffic in the multi-hop chain is converged to the root node $v_{0}$ via the wired interface. To forward the packets among these interfaces, the mesh node is designed as a layer 2 device by utilizing a bridge module.

Assume that the access location is $v_{k}$ for user $\mathrm{X}$, and the packets are relayed to prop-node $v_{j}$ step by step along the multi-hop chain $c<i, j>$ (let $i<j$ for simply illustration), then the packets will be transmitted and forwarded with n steps, where

$$
n=j-k+1
$$

\subsection{Latency Analysis}

Apparently the packet relay includes two processes as packet transmission between the neighbor nodes and packet forward among the internal interfaces. Let us denote that the transmission latency is $\tau_{k}^{a}$ and the forward latency is $\tau_{k}^{r}$, then the total latency for user $\mathrm{X}$ is

$$
\tau_{k}=\tau_{k}^{a}+\tau_{k}^{r}, i \leq k \leq j
$$

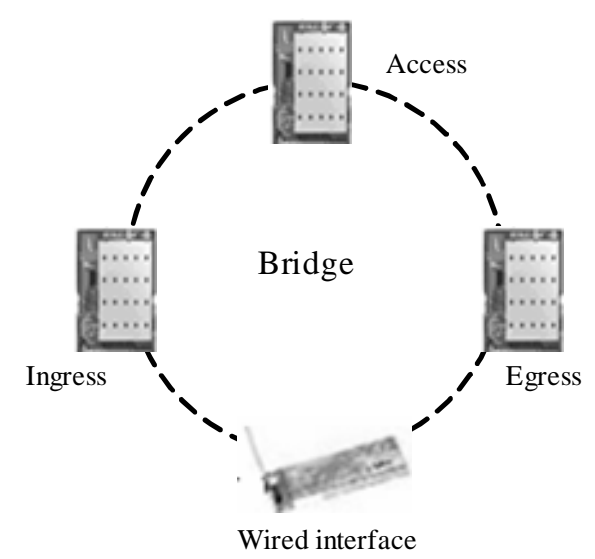

Figure 3. Mutlit-interface mesh node structure.
Because the mesh node should be in charge of the wireless access, ingress and egress packet forward, its media access control is more complex than the traditional network. Hence a concept of interval is introduced here to characterize the fluency of traffic, i.e., the waiting time for next transmission since user $\mathrm{X}$ has sent the current packet. Let us denote that the transmission interval is $t_{k}^{a}$ and the forward interval is $t_{k}^{r}$, then the total interval for user $\mathrm{X}$ is

$$
t_{k}=t_{k}^{a}+t_{k}^{r}, i \leq k \leq j
$$

Assume that the vectors of transmission and forward time of nodes in $c<i, j\rangle$ are $\left(T_{i}^{a}, T_{i+1}^{a}, \cdots, T_{j}^{a}\right)$ and $\left(T_{i}^{r}, T_{i+1}^{r}, \cdots, T_{j}^{r}\right)$, then the latency of user $\mathrm{X}$ to transmit the packets to prop-node $v_{j}$ is

$$
\tau_{k}=\sum_{s=k}^{j} T_{s}^{a}+\sum_{s=k}^{j} T_{s}^{r}, i \leq k \leq j
$$

because these tasks must be performed in serial.

As the contribution of multi-interface structure, the mesh nodes can perform the wireless access, ingress and egress transmission and packet forward in parallel. Hence the interval of user $\mathrm{X}$ to transmit the packets to prop-node $v_{j}$ is

$$
t_{k}=\max _{s=k}^{j}\left(T_{s}^{a}+T_{s}^{r}\right), i \leq k \leq j
$$

\subsection{Bandwidth Analysis}

Obviously the bandwidth of user $\mathrm{X}$ is restricted by the access link and multi-hop chain. Let us denote that the access, convergence and final bandwidth of user $\mathrm{X}$ are $\omega_{k}^{a}, \omega_{k}^{r}$ and $\omega_{k}$, then it has

$$
\omega_{k}=\min \left(\omega_{k}^{a}, \omega_{k}^{r}\right), i \leq k \leq j
$$

As the outcome of multi-interface mesh node structure, the multi-hop WMN has solved the bandwidth problem [14]. But as the backbone for all the users in the multi-hop chain, the node that is nearer to the prop-node should undertake more packets relay task. Assume that the vectors of access and convergence bandwidth are $\left(W_{i}^{a}, W_{i+1}^{a}, \cdots, W_{j}^{a}\right)$ and $\left(W_{i, i+1}^{r}, W_{i+1, i+2}^{r}, \cdots, W_{j-1, j}^{r}\right)$ within $c<i, j\rangle$, and the amount of user within $v_{k}$ and $c<i, j\rangle$ is $\lambda$ and $\kappa$ individually, then it has

$$
\begin{aligned}
& \omega_{k}^{a}=\frac{W_{k}^{a}}{\lambda} \\
& \omega_{k}^{r}=\frac{W_{j-1, j}^{r}}{\kappa}
\end{aligned}
$$


with a simplest and affair bandwidth assignment policy applied.

\section{Numerical Study}

In this section, we make an illustrative implementation proposal for a railway wireless coverage with $300 \mathrm{Km}$ distance. Assume that the coverage of each AP is $1 \mathrm{Km}$ and its fault probability is $0.1 \%$ independently, and it requires that

$$
\left\{\begin{array}{l}
\bar{\alpha} \leq 2, \quad 0.1 \% \leq o\left(V^{\prime}\right)<1 \% \\
\bar{\alpha} \leq 1.5, \quad o\left(V^{\prime}\right) \geq 1 \%
\end{array}\right.
$$

where $\bar{\alpha}$ is the average impact factor and $o\left(V^{\prime}\right)$ is the fault probability of set $V^{\prime}$.

Obviously, this test bed needs to deploy about 300 APs along the railway. To estimate the number of prop-nodes, we developed a simulation system with c programming language in Linux operation system to implement the factor $\alpha$ algorithm. In this simulation system, we assume that all the nodes are deployed evenly and their fault probabilities are equal and independently. Let the number of APs, i.e. the number of nodes, $|V|=301$. When the number of prop-nodes $\left|V^{*}\right|$ and fault nodes $\left|V^{\prime}\right|$ are $(4,6,7,11,16)$ and $(1,2,3)$ respectively, we obtained the distribution of $\bar{\alpha}$ as shown in Figure 4 .

Set the fault probability of every node as $0.1 \%$ and independently, then it has

$$
\left\{\begin{array}{l}
o\left(\left.V^{\prime}\right|_{\left|V^{\prime}\right|=0}\right)=73.997 \% \\
o\left(\left.V^{\prime}\right|_{\left|V^{\prime}\right|==}\right)=22.295 \% \\
o\left(\left.V^{\prime}\right|_{\left|V^{\prime}\right|=2}\right)=3.348 \% \\
o\left(\left.V^{\prime}\right|_{\left|V^{\prime}\right|=3}\right)=0.334 \% \\
o\left(\left.V^{\prime}\right|_{\left|V^{\prime}\right|>3}\right)=0.026 \%
\end{array}\right.
$$

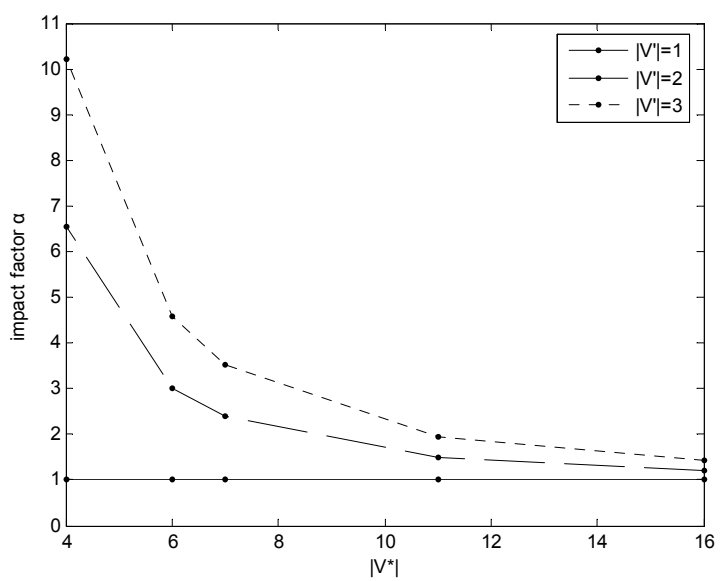

Figure 4. The distribution of average impact factor $\alpha$.
According to (11), the Equation (10) can be converted to

$$
\left\{\begin{array}{l}
\bar{\alpha} \leq 1.5,\left|V^{\prime}\right|=2 \\
\bar{\alpha} \leq 2, \quad\left|V^{\prime}\right|=3
\end{array}\right.
$$

Based on the distribution of $\bar{\alpha}$ with simulation, we can set $\left|V^{*}\right|=11$ to meet with the requirement of (10), i.e., deploy a prop-node every $30 \mathrm{Km}$.

As the most density of train running schedule in China, there has no more than 3 trains in the $30 \mathrm{Km}$ sector. As a typically bandwidth of WiFi for access and convergence, $W_{k}^{a}$ and $W_{j-1, j}^{r}$ may be deployed as 20Mbps and 60 Mbps [14], then the bandwidth for each train as $\omega_{k}=20 \mathrm{Mbps}$ according to Equations 8,9, which is more than the solutions with satellite and 2G/3G network technologies nowadays.

\section{Conclusions and Discussion}

Banyan-tree topology provides an alternative networking scheme for the ZWC with WiFi while the star topology requires too many cables and the bus topology is too weak. Numeric studies show that it only requires deploying 11 prop-nodes to wired network for a railway wireless coverage with $300 \mathrm{Km}$ distance, and the average impact factor $\bar{\alpha} \leq 2$ when the fault probability $0.1 \% \leq o\left(V^{\prime}\right)<1 \%$ and $\bar{\alpha} \leq 1.5$ when $o\left(V^{\prime}\right) \geq 1 \%$. At the same time, it can provide each train with 20Mbps bandwidth, which is more than the current solutions. Future work includes the capacity model for banyan-tree topology based ZWC and a dynamical fault probability detection scheme.

\section{Acknowledgments}

The work was supported financially by the Fujian Provincial Department of S\&T through the project AEED (No. 2008-80) and Wi-Road (No. 2009-27).

\section{References}

[1] China Internet Network Information Center, the 24th Statistical Report on Internet Development [R], Beijing, China, July 2009.

[2] Ministry of Tranport of the People's Republic of China, Statistical Communique on the 2008 Road and Waterway Development [R], Beijing, China, April 2009.

[3] Ministry of Railways of the People's Republic of China, Statistical Communique on the 2008 Railway Development [R], Beijing, China, June 2009.

[4] X. H. Jiang, F. M. Zou, Z. X. Lin, and T. S. Wang, “A Survey on the Internet Application on Passenger Train 
[J],” Journal of the China Railway Society, Vol. 29, No. 5, pp. 103-110, 2007.

[5] Roamad, "Highway Wi-Fi networks: A RoamAD whitepaper with a case study on the Arizona I-19 highway WiFi corridor [R],” February 2006, http:/www.kordiasolutions.com/files/RoamAD_Case_St udy_Arizona_Highway_Wi-Fi_Corridor.pdf.

[6] Y. F. Ko, M. L. Sim, and M. Nekovee, "Wi-Fi based broadband wireless access for users on the road [J]," BT Technology Journal, July 2006.

[7] U. S. Department of Transportation, Federal Railroad Administration, Study of High-Speed Wireless Data Transmissions for Railroad Operation [R], RR07-10, U.S.A, April 2007, http://www.fra.dot.gov/downloads/Research/rr0710.pdf.

[8] S. Savary and A. De Cort, "WiFi services in fast speed trains: The thalys user experience [C],” Train Communications 2008, London, England, June 2008.

[9] G. I. Ohta, F. Kamada, N. Teramura, et al., "5 GHz WLAN verification for public mobile applications-Internet newspaper on train and advanced ambulance car [C],” In proceedings of the 1st IEEE Consumer Commu- nications and Networking Conference, pp. 569-574, 2004.

[10] M. Hempel, H. Sharif, Z. Ting, et al., "A wireless test bed for mobile 802.11 and beyond [C]," International Wireless Communications and Mobile Computing Conference, Vancouver, Canada, pp. 1003-1008, 2006.

[11] A. Stefan and S. Wolfgang, "Performance measurements in wireless 802.11g Multi-Hop Networks [D]," the University of H“'ogskolani Halmstad, Sweden, May 2006.

[12] R. D. Chen, H. W. Li, F. H. Li, et al., "Performance optimization in wireless Mesh networks based on Mesh point priority mechanism [C]," In Proceedings of Asia-Pacific Advanced Network, Singapore, 2006.

[13] Knuth and E. Donald, "The art Of computer programming: Fundamental algorithms, 3rd Edtion [M],” Addison-Wesley, Boston, Vol. 1, 1997.

[14] F. M. Zou and X. H. Jiang, "Improve the capacity of wireless backbone in multi-hop mesh network [J]," Science Technology and Engineering, Vol. 8, No. 13, pp. 3678-3681, 2008, 